\title{
La escuela y los alumnos con retraso mental
}

\author{
Josep Font
}



En las lineas que siguen, se aborda la problemática específica de los niños con retraso mental desde un enfoque sistémico que contemple a estos niños como sujetos con capacidades interactivas en las que debe basarse la escolarización.

\section{PRESENTACION}

Un sistema educativo inmerso en un período de intensa reflexión y reforma posibilita enfoques nuevos y diferentes aunque impone, también, ciertas cautelas y precauciones. Eso es así en todos los sectores y ámbitos educativos pero resulta particularmente cierto cuando nos referimos a los alumnos con necesidades educativas especiales. Más concretamente, la educación de los niños y adolescentes con condiciones de retraso mental supone un reto importante para el actual sistema educativo. Dilucidar cuáles son las mejores prácticas educativas, así como los estándares de una educación adecuada y de calidad para los alumnos con retraso mental, no es tarea fácil. Proponer enfoques que respondan al marco educativo actual y a las sugerencias de la literatura e investigación científica será un trabajo obligado para los profesionales'de este campo.

En el momento actual, somos conscientes de que la educación de los alumnos con retraso mental no se compone exclusivamente de buenas prácticas, de estrategias adecuadas o de la aplicación de las nuevas tecnologías. Una buena actuación parte de supuestos teóricos y de principios filosóficos claros y definidos que cualquier sistema educativo debe asumir e impulsar. Una educación de calidad para los alumnos con n.e.e. no sólo es un problema pedagógico o legislativo, es también un problema de valores (Gartner y Lipsky, 1987).

No es finalidad de este trabajo realizar una revisión exhaustiva de los supuestos en los que debe basarse la educación de los alumnos con retraso mental; no obstante, me gustaría aportar algunas sugerencias claves que constituyen elementos esenciales en los planteamientos educativos que podamos y debamos formular.

En primer lugar, la educación de los alumnos con retraso mental se tiene que abordar desde la óptica y la perspectiva de que el sistema educativo vigente afronte el tratamiento de la diversidad. Los alumnos con retraso mental requieren una educación acorde con sus características y necesidades, pero no al margen de los princi- 
pios, finalidades y recursos que el sistema escolar proporciona a los demás alumnos. La LOGSE asume claramente los principios básicos en que debe basarse la educación de los alumnos con n.e.e. y presenta un modelo educativo decididamente integrador y comprensivo. No obstante, un marco legal es una condición necesaria pero no suficiente para una educación adecuada y de calidad. Remodelar y cambiar los esquemas, las organizaciones y las prácticas viejas será un trabajo lento y costoso. La cultura profesional y la organización escolar en referencia a la educación de los alumnos con n.e.e. requieren modificaciones significativas para adaptarse a las nuevas líneas y paradigmas (Skrtic, 1991).

Dentro de este contexto hay tres elementos que me parecen fundamentales y que, tanto en el momento actual como en el futuro, van a determinar el tipo de educación que los alumnos con retraso mental van a recibir. Nos referimos a la escuela y a su organización, a la percepción que tengamos del alumno y concretamente del alumno con retraso mental y de la educación que precisa.

Parece obvio que debamos dirigirnos hacia un tipo de escuela cuya función principal es la educación de todos los alumnos, sean las que sean sus necesidades, características y circunstancias personales. Sólo así podemos conseguir un único sistema educativo y de calidad.

Necesitamos un escuela comprensiva e inclusiva, en la que se proporcione a cada uno de los alumnos programas educativos adecuados que se adapten a sus características particulares, así como al tipo de apoyo y ayuda que ellos o sus maestros puedan precisar (Stainback y Stainback, 1990; Stainback y Stainback, 1992).

Para conseguir esta finalidad, la escuela debe adquirir una nueva mentalidad, debe plantear una organización y estructuración más flexibles, disponer de materiales y currículos adaptados y de los recursos convenientes.

Además, importa una visión renovada de los alumnos con retraso mental y de la educación que se les debe proporcionar. En el momento actual estamos viviendo cambios sustanciales en el concepto de retraso mental que van a suponer modificaciones altamente significativas en la forma de entender y prestar los servicios (AAMR, 1992; Coulter, 1991; Luckasson et al., 1992). La nueva definición que propone la "American Association on Mental Retardation", así como los supuestos que asume, propician una visión del retraso mental radicalmente distinta. En ella se intenta observar el nivel de funcionamiento de una persona dentro del contexto de su entorno (interacción entre capacidades, nivel de funcionamiento y entornos) y la clasificación se basa en la intensidad de apoyos necesarios más que en el grado de retraso derivado de un CI. De esta forma, ya no podemos percibir a los alumnos con retraso mental como individuos aislados y sujetos de múltiples etiquetas y categorías, sino como personas potencialmente activas de una sociedad que merecen nuestro respeto y apoyo. Podemos afirmar que el retraso mental representa la interacción de circunstancias personales y sociales, en las que las variables de apoyo alteran sustancialmente el nivel de funcionamiento de la persona.

Finalmente, debemos superar una educación que se centraba básicamente en la atención de los déficits individuales e ir hacia la provisión de un sistema de apoyos adecuados. Además de garantizar el desarrollo individual del alumno, debemos observar las interacciones existentes entre éste y el entorno y, de esta forma, derivar las ayudas, los apoyos y los recursos que necesita para una adaptación y funcionamiento óptimos.

Parece obvio que la naturaleza de los cambios aquí propuestos significarán variaciones en las prácticas profesionales y en las organizaciones escolares. No obstante, debemos afrontar los cambios con una clara visión de futuro. El análisis de la situación actual nos hace predecir unas perspectivas en estas direcciones (Ludlow y Lombardi, 1992). 


\section{VALORACION DE LAS NECESIDADES EDUCATIVAS}

La introducción del concepto de necesidades educativas ha supuesto enfoques distintos en la evaluación de los alumnos con retraso mental. Las necesidades educativas y las necesidades educativas especiales tienen que entenderse dentro de un continuum de grados diferentes y que son relativas tanto a los factores internos del niño como a las disponibilidades de recursos adecuados en su entorno (Wedell, 1991).

Hay que enfatizar la idea del continuum que presuponen las necesidades educativas, que van de las más generales a las más específicas. Asimismo, debemos remarcar su grado de relatividad, tanto en relación al marco en que se definen como en el contexto en que se evalúan. En este sentido, debe prestarse atención también al carácter interactivo de las necesidades. Estas no dependen sólo de las características personales del alumno, sinó también de las características del entorno educativo y familiar en el que se desarrolla.

Es a partir de estos supuestos que emprendemos la valoración de los alumnos con retraso mental y no a partir de criterios diagnósticos o clasificatorios que aportan poca información sobre los medios y recursos que un alumno va a precisar a lo largo de su escolarización.

La identificación, determinación y valoración de las necesidades educativas es un proceso sistemático de análisis y recogida de información relevante para tomar decisiones educativas. Tiene que permitir la toma de decisiones en relación a: a) qué, cómo y cuándo enseñar, b) tipo de servicios específicos que el alumno precisa y c) emplazamiento del alumno (Giné et al., 1989).

En la valoración de las necesidades educativas de los alumnos con retraso mental, el currículum ordinario constituye el elemento básico de referencia. Determinar lo que el alumno precisa en base a su nivel de competencias y a las características de su entorno escolar y familiar, así como de los recursos personales y materiales que van a ser imprescindibles, es una de las finalidades básicas de este proceso. No obstante, debemos observar que los alumnos con retraso mental, especialmente los que presentan las condiciones más graves y permanentes, pueden mostrar necesidades que sobrepasen el estricto marco curricular y/o incluyan otras necesidades que requieran también valoración. Nos referimos a aquellas necesidades derivadas de áreas que en muchos casos no están incluidas en el currículum escolar (especialmente las áreas relativas a favorecer el funcionamiento independiente en la comunidad) así como a aquellos aspectos vinculados a factores personales (necesidades socio/emocionales y de tipo médico). Aun así, debemos insistir en que, en la etapa educativa, el currículum ordinario debe representar el elemento esencial en la valoración de las necesidades educativas. Es en este sentido que adquieren valor $\mathrm{e}$ importancia las evaluaciones contextuales (Browder, 1987; Gaylord-Ross y Browder, 1991; Juan-Espinosa et al., 1992; Neel y Billingsley, 1989). Los sistemas de evaluación o medida en base al currículum están constituyendo un enfoque nuevo y prometedor en la evaluación de necesidades (Cohen y Spruill, 1990, Shinn, 1989).

Para la recogida de datos e información debemos utilizar distintos instrumentos y estrategias. Entre otros podemos citar los sistemas de observación directa, los cuestionarios, las listas o escalas de verificación, los inventarios, las muestras de trabajo, las entrevistas, los sistemas formales (pruebas estandarizadas de rendimiento, escalas de conducta adaptativa, etc.).

Desgraciadamente disponemos de pocos sistemas de toma de decisiones para la valoración de las necesidades y de insuficientes instrumentos útiles y válidos. A pesar de las dificultades inherentes a todo proceso de valoración y de las carencias existentes, deberíamos tener claro que, a partir de la evaluación de los alumnos con retraso mental, importa obtener la siguiente información: 
a) Una descripción de los niveles actuales de competencia en relación a las áreas del currículum escolar.

b) Una descripción de los factores que facilitan o dificultan el aprendizaje.

c) Una descripción de los puntos fuertes y débiles del alumno.

d) Un análisis descriptivo de las posibles relaciones existentes entre:

- las habilidades en una área en relació a possibles déficits en otra.

- las dificultades en las habilidades previas, estrategias de aprendizaje, etc.

- la historia previa de escolarización.

- los factores de tipo médico, social y cultural.

e) La identificación y determinación de las necesidades.

f) El tipo de servicios específicos.

h) El emplazamiento del alumno.

En último lugar, todo proceso de identificación y valoración de las necesidades debe exigir la participación de todos los profesionales que comparten la acción educativa de los alumnos. Los padres también forman parte importante en este proceso de valoración.

\section{CRITERIOS PARA SU ESCOLARIZACION}

Los alumnos con retraso mental constituyen un grupo de alumnos muy heterogéneo. Aunque comparten ciertos atributos comunes, las características y el grado y tipo de necesidades pueden variar enormemente de un alumno a otro. Por este motivo, los criterios de escolarización pueden llevar a soluciones distintas para unos y otros y tendrán en cuenta tanto las condiciones personales como las disponibilidades de recursos de su entorno.

El artículo 37 de la LOGSE establece que «la educación en unidades o centros de educación especial se llevará a cabo cuando las necesidades del alumno no puedan ser atendidas por un centro ordinario». Así pues, y de acuerdo con la legislación actual, la escolarización de los alumnos con retraso mental se efectuará, siempre que sea posible y que las circunstancias lo permitan, en centros ordinarios. Las unidades y centros de educación especial escolarizarán a aquellos alumnos con las necesidades más graves y permanentes (por lo general alumnos con retraso mental severo y profundo). Debemos insistir en este proceso progresivo de integración e inclusión de los alumnos con retraso mental en la escuela ordinaria y orientar su escolarización en unidades o centros de educación especial sólo cuando se presenten las necesidades más graves.

Cualquiera que sea el tipo de escolarización que se haya acordado para un alumno, esta situación debe ser evaluada regularmente para favorecer, en los casos que sea posible, el acceso a un régimen de mayor integración.

No obstante, las condiciones personales y de handicap no deben representar los únicos criterios para su escolarización. El emplazamiento escolar de un alumno debe ser el resultado de la valoración de sus necesidades en relación con el currículum escolar y los recursos materiales y humanos disponibles en su entorno, dando un énfasis especial a los del entorno más ordinario. Estos deben ser los criterios básicos para decidir la escolarización de los alumnos.

\section{OBJETIVOS Y CONTENIDOS PRIORITARIOS DURANTE LA ESCOLARIZACION OBLIGATORIA}

Entre los alumnos con n.e.e., quizás el grupo de alumnos con retraso mental sea uno de los que exijan las adecuaciones más particulares del currículum y, en con- 
secuencia, de los contenidos y objetivos educativos. No obstante, no nos parece oportuno abordar este tema desde una perspectiva exclusiva de la especificidad, sino a partir de los elementos comunes que el sistema educativo ofrece a todos los alumnos.

Hasta hace poco, el sistema escolar prefería proporcionar un sólido conocimiento y unas competencias básicas en las áreas consideradas fundamentales. A medida que la sociedad se ha hecho más compleja y que los conocimientos han aumentado brutalmente, las finalidades de la educación han variado de manera sustancial. Las características de nuestro mundo actual cambiante e interdependiente hace difícil la declaración de unas finalidades educativas concretas.

Así pues, debemos ser conscientes de las finalidades amplias y globales de la educación (Ford, Davern y Schorr, 1992). En esta dirección, no resulta extraño afirmar que las finalidades de la educación son iguales para todos los alumnos, es decir, conseguir el desarrollo máximo de sus capacidades para poder vivir y trabajar de la forma más independiente posible en la sociedad. Podemos observar cómo algunas de las finalidades que el actual sistema educativo propone para las distintas etapas de la educación obligatoria pueden ser aplicadas a los alumnos con retraso mental. Y no sólo pueden ser aplicadas, sino que sería importante que todos los alumnos pudieran trabajar o que en realidad se orientasen a la consecución de los mismos resultados amplios de la educación.

Conviene matizar, en este punto, que lo que diferirá es el nivel en que estas finalidades se podrán conseguir (es decir, el grado y tipo de aprendizaje) y el énfasis que se ponga en cada una de ellas. De acuerdo con la edad, las necesidades que se presenten, las características del entorno, el tipo de emplazamiento, etc., propondremos unas u otras finalidades y pondremos mayor énfasis en unas áreas determinadas o en otras. Asimismo, esta opción no es incompatible con que haya contenidos y objetivos diferentes para algunos alumnos ni que las finalidades globales se puedan concretar en resultados más específicos. Es en relación a los resultados comunes de la educación que se deben adaptar, introducir o añadir nuevos contenidos o establecer prioridades distintas.

Intentamos trabajar en base a lo que tienen en común y a lo que tienen de específico los alumnos y no sólo partiendo de este último aspecto. Sabemos que los alumnos con retraso mental exigen adecuaciones, modificaciones y ampliaciones del currículum escolar y que, en algunos casos, éstas van a resultar sustancialmente precisas o diferentes. No obstante, esto no contradice que puedan participar, en lo que sea posible, de las experiencias y oportunidades comunes a todos los alumnos.

A pesar de las consideraciones anteriores, pensamos que los objetivos y contenidos prioritarios para los alumnos con retraso mental durante la escolarización obligatoria pueden concretarse en los siguientes:

1. La adquisición de los hábitos de autonomía personal y social, de las habilidades de relación e interacción que permitan el acceso a los entornos y servicios comunitarios de la forma más independiente posible.

2. Potenciar el desarrollo de las posibilidades comunicativas (a través del lenguaje oral o de algún sistema alternativo).

3. Favorecer el desarrollo de aquellas habilidades, destrezas, y valores que garanticen el desarrollo personal y/o puedan ser necesarios para el desarrollo de otras habilidades.

4. Facilitar la adquisición de habilidades académicas generalizadas (lectura, escritura, cálculo) o, en su caso, de habilidades académicas funcionales.

5. Promover los conocimientos y valores básicos que permitan la comprensión y acceso a su entorno y a la cultura.

6. Potenciar las habilidades y la ocupación significativa en actividades de ocio y tiempo libre. 
7. Proporcionar la formación y orientación adecuadas para la preparación al mundo del trabajo y a la vida adulta.

Como resumen podemos decir que los objetivos y contenidos educativos tiene que ser relevantes, significativos, funcionales, adecuados a la edad cronológica, en base a los entornos naturales y que promuevan la participación activa del alumno.

\section{ESTRATEGIAS METODOLOGICAS Y ORGANIZATIVAS MAS FAVORABLES}

Una educación adecuada debe coordinar de forma efectiva el conjunto de elementos que en ella confluyen. No existe una sola estrategia metodológica ni organizativa que responda mejor a las necesidades de los alumnos con retraso mental. Es a partir del ajuste educativo que requiere cada alumno en relación con su entorno y al tipo de aprendizajes que necesita, que vamos a proponer las estrategias metodológicas y organizativas más apropiadas. En la actualidad disponemos de un buen número de estrategias que han demostrado su eficacia y eficiencia (Falvey, 1986; Langone, 1986; Matson y Mulick, 1983; Polloway et al. 1985; Snell, 1987).

Los alumnos con retraso mental, en la mayoría de los casos, no aprenden con los mismos procedimientos y estrategias que los alumnos normales. Así pues, las modificaciones organizativas, la planificación de todo el proceso educativo, el uso de procedimientos de intervención probados así como de las ayudas y adaptaciones necesarias resultarán fundamentales para garantizar su progreso personal y social.

De esta forma, es en la mayoría de casos imprescindible planificar de forma precisa el conjunto de actuaciones educativas dirigidas a los alumnos con retraso mental.

Sabemos que el entorno educativo tiene más influencia sobre los alumnos discapacitados que sobre los que no presentan dificultades (Gotllieb et al., 1991). Aunque el tipo de escolarización va a ser un factor relevante en la utilización de ciertas estrategias, las siguientes pueden considerarse como las más favorables e importantes:

a) Estrategias organizativas. Nos referimos aquí a las modificaciones, tanto generales a la escuela como específicas a la clase, que pueden favorecer el proceso de enseñanza y aprendizaje de los alumnos: los criterios de agrupamiento, la organización de grupos flexibles o alternativos, las posibilidades de trabajo individual, etc...

b) Estrategias y procedimientos instruccionales. Existe un número elevado de técnicas y procedimientos instruccionales para la adquisición y mantenimiento de nuevas habilidades así como para hacer frente a los problemas de comportamiento. Podemos citar las estrategias de ayuda y control de estímulos, los procedimientos basados en el entorno (enseñanza incidental, Time Delay, Mand-Model) y los procedimientos de creación de nuevos repertorios (modelamiento, encadenamiento). Asimismo, y a medida que los alumnos con retraso mental se han integrado en las escuelas ordinarias, han aparecido un nuevo conjunto de estrategias que facilitan la interacción y el aprendizaje de estos alumnos junto a sus compañeros de clase (Stainback y Stainback, 1990). En este sentido podemos hablar de enseñanza a través de los compañeros, aprendizaje cooperativo, equipos de apoyo, enseñanza en equipo, etc. De la misma forma, y para hacer frente a los problemas de comportamiento que algunos alumnos pueden presentar, se han desarrollado últimamente estrategias de programación positiva que aportan un enfoque nuevo y prometedor en este campo (LaVigna, Willis y Donellan, 1989).

c) Estrategias de generalización y de intervención comunitaria. Una de las características básicas de los alumnos con retraso mental es que no generalizan las habilidades aprendidas a distintas situaciones, lugares y personas. Lógicamente, no 
podemos enseñar cada una de las habilidades en todas las situaciones y personas posibles. El uso de contextos naturales, de situaciones y materiales reales y de técnicas de generalización es imprescindible para conseguir unos resultados significativos (Horner, Dunlap y Koegel, 1988). En esta misma línea, se han desarrollado estrategias de intervención comunitaria para mejorar el nivel de adquisición de habilidades funcionales en entornos integrados (Sailor et al., 1988; Snell y Browder, 1986; Westling y Floyd, 1990).

e) Estrategias cognitivas. En la actualidad se pone más énfasis en los procesos de aprendizaje que en los resultados finales. En el campo de la educación han adquirido una gran importancia las estrategias cognitivas. Si bien su aplicación a la educación de los alumnos con retraso mental es escasa, sí que resultan un campo abierto y de muy reciente implantación (Ashman y Conway, 1989; Meyen, Vergason y Whelan, 1989).

f) Uso de las nuevas tecnologías. Parece lógico pensar que el potencial que supone el uso de los ordenadores, los nuevos avances en robótica y los progresos médicos pueden afectar la educación de los alumnos con retraso mental (Ludlow y Lombardi, 1992).

\section{CRITERIOS Y PROCEDIMIENTOS PARA LA EVALUACION}

La evaluación del progreso de los alumnos es parte integrante del mismo proceso educativo. En este sentido, la evaluación tiene una función antes, durante y después del proceso de enseñanza-aprendizaje. No debemos confundir la evaluación con la práctica de pasar test o realizar exámenes. La evaluación incluye todos los métodos disponibles de recogida de información en relación a la educación del alumno y a la eficacia del maestro. El proceso de evaluación debe ser contemplado en el contexto de los objetivos identificados para un alumno concreto y tiene que aportar información, como mínimo, sobre los siguientes aspectos: a) si se han conseguido los objetivos, b) la eficacia del programa educativo y del proceso de enseñanza y c) las modificaciones y el tipo de ajustes a realizar.

La evaluación debería ir más allá de la simple medida del rendimiento académico de los alumnos. Los siguientes criterios (Cullen y Pratt, 1992) pueden asegurar una evaluación adecuada y como parte inherente al proceso de enseñanza y aprendizaje:

a) La evaluación continuada del rendimiento del alumno es una parte integral del proceso de enseñanza-aprendizaje y debería identificar rápidamente las dificultades y proporcionar una base para la acción inmediata.

b) La forma de evaluación debería ser adecuada a la tarea, la clase y etapa de aprendizaje.

c) El efecto de la evaluación debe ser constructivo. Las técnicas de evaluación deben aumentar en el alumno, y no disminuir, el sentimiento del esfuerzo personal y de lo que es importante en su aprendizaje.

d) Se deben utilizar una variedad de técnicas evaluativas a fin de obtener información sobre diferentes aspectos del aprendizaje del alumno, que proporcionen un fundamento fiable para las opiniones y decisiones.

e) La evaluación debe ir más allá de las áreas cognitivas y psicomotoras e introducir el dominio afectivo que incluya una exploración de los intereses, valores y actitudes.

f) Para que la evaluación sea efectiva, debe haber comunicación en muchas direcciones (alumno-maestro, maestro-alumno, maestro-padres, etc.).

g) El proceso de evaluación debería ayudar a los alumnos a aprender cómo evaluar su propio aprendizaje. 
Cuando se han identificado los propósitos de la evaluación y se han determinado los objetivos a evaluar, podemos utilizar distintos procedimientos y estrategias que se adapten a ellos. Entre las estrategias más importantes podemos señalar: a) medidas de productos permanentes (frecuencia, porcentajes, número de ensayos, análisis de tareas), b) registros de observación directa, c) revisión de los registros acumulativos, d) entrevistas con el alumno, e) pruebas informales, f) pruebas elaboradas por el maestro, $g$ ) autoevaluaciones y $h$ ) pruebas estandarizadas.

Recoger información tiene poca utilidad si no se utiliza para tomar decisiones que mejoren el proceso de enseñanza y aprendizaje. El factor primordial para una evaluación signifi-cativa es aprender a leer e interpretar los datos. Por este motivo, se debe disponer de procedimientos de recogida de datos así como de guías para la toma de decisiones (Browder, 1987).

\section{Referencias}

AAMR (1992). AAMR board approves new MR definition. AAMR News $\varepsilon$ Notes, vol. 5, $n^{\circ} 4,1 / 6$.

Ashman, A.F. y Conway R. (1990). Estrategias cognitivas en educación especial. Madrid: Aula XXI, Santillana.

Browder, D. (1987). Assessment of individuals with severe handicaps: An applied babavior approach to life skills assessment. Baltimore: Paul H. Brookes.

Cohen, L. G., y Spruill, A. (1990). A practical guide to curriculum riculum-based assessment for special educators. Illinois: Charles C. Thomas Publisher.

Coulter, D. L. (1991). The failure of prevention. Mental Retardation, vol. 29, $n^{\circ} 5,3-4$.

Cullen, B., y Pratt, T. (1992). Measuring and reporting student progress. En W. Stainback y S. Stainback, Curriculum considerations in inclusive classmoms. Facilitating learning for all students (pag. 175-196). Baltimore: Paul H. Brookes.

Falvey, M. A. (1986). Community-based instruction. Instructional strategies for students with severe handicaps. Baltimore: Paul H. Brookes.

Ford, A.; Davern, L., y Sshorr, R. (1992). Inclusive education: "Making sense" of the curriculum. En W. Stainback y S. Stainback, Curriculum considerations in inclusive classrooms. Facilitating learning for all students (pag. 37-61). Baltimore: Paul H. Brookes.

GAYLORD-ROSs, R., y BROWDER, D. (1991). Functional assessment. Dynamic and domain properties. En L. H. Meyer, C.A. Peck y L. Brown (Eds), Critical issues in the lives of people with severe disabilities (pag. 45-66). Baltimore: Paul H. Broo-kes.

GARTNER, A., y LIPSKY, D. K. (1987). Beyond special education: Toward a quality system for all students. Harvard Educational Review, vol. 57. $n^{\circ} 4,367-395$.

Giné, C.; Carasa, P.; Fernandez, M.; Madurell, J.; Ruiz, R., y Tirado, V. (1989). Educació especial. Noves perspectives. Barcelona: Editorial Laia.

GotTLIEB, J.; ALTER, M., y GOTLLIEB, B. W. (1991). Mainstreaming academically handicapped children in urban schools. En J.W. Lloyd y N.N. Singh (Eds), The regular education iniciative: Alternative perspectives on concepts, issues and methods (pag. 95-112). Dekalb, IL: Sycamore

HORNER, R. H.; DuNLAP, G., y KOEGEL, R. L. (1988). Generalization and Maintenance. Liff-Style changes in applied seyyings. Baltimore: Paul $\mathrm{H}$. Brookes.

JuAN-ESPINOSA, M.; COLOM, R., y FloRes, V. (1992). Contexto y retraso mental. Madrid: Ministerio de Educación y Ciencia.

LANGONE, J. (1986). Teaching retarded learners. Curriculum and methods for improving instruction. Boston: Allyn and Bacon.

LAVIGNA, G.; WILUS, T., y DONNELLAN, A. (1989). Tue role of positive programming in behavioral treatment. En E. Cipani (Ed), The treatment of severe bahaviors disorders. Behavior analysis approaches, (pag. 59-83). Washington: American Association on Mental Retardation.

LuCKasson, R. et al., (1992). Mental Retardation. Definition, Classification and Systems of Supports. Washington: American Association on Mental Retardation.

LUDLOW, B. L., y LOMBARDI, T. P. (1992). Special education in the year 2000: Current trends and future developments. Education and Treatment of Children, vol. 15, $n^{\circ} 2,147-162$.

MATson, J. L., y MuLICK, J. A. (1983). Handbook of mental retardation. New York: Pergamon Press.

MEYEN, E.; VERGASON, G., y WHELAN, R. (1988). Effective instructional strategies for exceptional children. Denver: Love Publishing Company.

NEEI, R. S., y BUUNGSIEY, F. (1989). IMPACT. A functional curriculum bandbook for students with moderate to severe disabilities. Baltimore: Paul $\mathrm{H}$. Brookes. 
Polloway, E.; PAYNe, J ; PATtON, J., y PAYNE, R. (1985). Strategies for teaching retarded and special needs leamers. Columbus: Charles E. Merril.

SAILOR, W.; GOETZ, L.; ANDERSON, J.; HUNT, P., y GEE, K. (1988). Research on community intensive instruction as a model for building functional generalized skills. En R.H. Horner, G. Dunlap y R.L. Koegel (Eds), Generalization and Maintenance. Life-Style changes in applied seyyings. Baltimore: Paul H. Brookes.

SHINN, M. R. (1989). Curriculum-based measurement. Assessing special children. Niew York: The Guilford Press.

SKRTIC, T. M. (1991). Bebind special education. A critical analysis of professional culture and school organization. Denver: Love Publishing Company.

SNELI, M. E. (1987). Systematic instruction of persons with severe bandicaps. Columbus: Charles E. Merrill.

SNELL, M. E., y BROWDER, D. (1986). Community-referenced instruction: Research and issues. The Joumal of Association with Severe Handicaps, vol. 11, $n^{\circ} 1,1-11$

STAINBACK, W., y STAINBACK, S. (1990). Support networks for inclusive schooling, Interdependent integrated education. Baltimore: Paul H. Brookes.

StaINBaCK, W., y StaINBaCK, S. (1992). Curriculum considerations in inclusive classrooms. Facilitating learning for all students. Baltimore: Paul $\mathrm{H}$. Brookes.

WEDEL, K. (1991). The national curriculum and special educa- tional needs. En D. Lawton y C. Chitty (Ed.), The National Curriculum (pag. 102-112). London: Institute of Education, University of London.

WeSTLING, D. L., y FloYd, J. (1990). Generalization of community skills: How much training is necessary?. The Journal of Special Education, vol. $23, n^{\circ} 4,386-406$.

\section{Bibliografía básica. Sugerencia para los lectores}

ASHMAN, A. F., y CONWAY, R. (1990). Estrategias cognitivas en educación especial. Madrid: Aula XXI, Santillana.

Brechin, A., y Swan, J. (1990). Cambio de relaciones. Bar-celona: Ediciones Milán.

BrenNan, W. K. (1988). El currículo para niños con necesidades especiales. Madrid: M.E.C. y Siglo XXI.

BROWN, L. (1989). Criterios de funcionalidad. Barcelona: Edi-ciones Milán

Centro Nacional de ReCursos para la Educacion Especial, (1991). El alumno con retraso mental en la escuela ordinaria. Ma-drid: Ministerio de Educación y Ciencia.

Donlon, E. T., y FulTon-Burtonb, L. (1989). La enseñanza de los deficientes severos y profundos. Madrid: M.E.C. y Siglo XXI.

Gine, C.; Carasa, P.; Fernandez, M.; Madurell, J.; Ruiz, R., y Tirado, V. (1989). Educació especial. Noves perspectives. Barcelona: Editorial Laia.

INGALLS, R. P. (1982). Retraso mental. La nueva perspectiva. México Editorial El Manual Moderno.

LOVAAS, I. (1990). Enseñanza de niños con trastornos del desa- rrollo. Barcelona: Martínez Roca

Ruiz, R. (1988). Técnicas de individualización didáctica. Ade- cuaciones curriculares individualizadas para alumnos con necesidades especiales. Madrid: Editorial Cincel. 


\section{La escuela y los alumnos con retraso mental Josep Font CL\&E, 1994, 22, pp. 37-46}

Resumen: El presente trabajo intenta abordar la educación de los alumnos con retraso mental durante la escolarización obligatoria. Parte de unos supuestos básicos en relación a la escuela, al alumno y a la educación. El concepto de escuela y su orga-nización, la percepción del alumno y concretamente del alumno con retraso mental y de la educación que precisa van a determinar de forma decisiva el tipo de escolarización y educación que se va a prestar. Posteriormente y en base a los puntos an-teriores se analizan aquellos componentes esenciales que deben tenerse en cuenta en el proceso de escolarización de estos alumnos. Así pues, la valoración de las necesidades educativas, los criterios para su escolarización, los objetivos y contenidos prioritarios, las estrategias metodológicas y organizativas más favorables y los criterios y procedimientos para la evaluación son elementos básicos que se definen para garantizar una educación adecuada y de calidad..

Datos sobre el autor: El autor es pedagogo, director del CPT l'Estel y profesor de l'Escola universitària de Mestres "Balmes", Estudis Universitaris de Vic.

Dirección: C.E.E. «L'Estel». Finca St. Tomás, s/n. 08519 Caldetenes. Barcelona.

(C) PERMISOS PARA CITAR O REPRODUCIR EN OTRAS FUENTES: Se pueden citar libremente hasta 500 palabras. Para reproducir una porción de texto mayor, figuras o ilustraciones, se deberá pedir permiso por escrito a la revista, especificando el uso al que se destina el texto. En todos los casos, se deberá citar el copyright de $C L \& E$. En el caso de artículos o textos que hayan sido a su vez reproducidos en $C L \& E$ los interesados deberán dirigirse tanto a los detentadores del copyright original como a $C L \& E$, en el caso de que se quiera hacer uso de la traducción. FOTOCOPIAS: Para todo lo relacionado con el uso mediante fotocopia del material de esta revista, deberán dirigirse a: CEDRO, C/ José Marañón, 10, 3. Izda. Tel. 5941575 . Fax 4453567 